\title{
Black holes, the van der Waals gas, compressibility and the speed of sound
}

\section{Talk presented at the 13th Hellenic School and Workshops on Elementary Particle Physics and Gravity, 31 Aug.-15 Sep. 2013, Corfu, Greece}

\author{
Brian P. Dolan ${ }^{1,2, *}$ \\ ${ }^{1}$ Department of Mathematical Physics, National University of Ireland Maynooth, Ireland \\ ${ }^{2}$ School of Theoretical Physics, Dublin Institute for Advanced Studies, Dublin, Ireland
}

Received and revised 3 March 2014, accepted 6 March 2014

Published online 5 May 2014

Key words Black hole, thermodynamics, anti-de Sitter space-time, compressibility.

We review some recent developments in black hole thermodynamics for asymptotically anti-de Sitter black holes. Viewing the cosmological constant as a thermodynamic pressure allows a thermodynamic volume to be defined, the thermodynamically conjugate variable to the pressure. The properties of the equation of state can then be studied and a compressibility and a speed of sound is defined for such black holes.

(C) 2014 WILEY-VCH Verlag GmbH \& Co. KGaA, Weinheim

\section{Review of black hole thermodynamics}

Over 40 years ago Bekenstein [1] argued that a black hole should have an entropy associated with it, proportional to the area $A$ of the event horizon. In Planck units, with $\ell_{P l}^{2}=\hbar G / c^{3}$ and $G=c=1$,

$$
S \propto \frac{A}{\ell_{P l}^{2}} .
$$

This opened the door to the whole subject of black hole thermodynamics, as an entropy suggests a temperature. Hawking subsequently calculated the temperature [2] in terms of the surface gravity $\kappa$ of the black hole and found

$$
T=\frac{\kappa \hbar}{2 \pi} .
$$

For a Schwarzschild black-hole in four dimensions, for example, $\kappa=\frac{1}{4 M}$ giving

$$
T=\frac{\hbar}{8 \pi M}
$$

In thermodynamics the temperature is related to the internal energy $U(S)$ by $T=\frac{\partial U}{\partial S}$ and, identifying this with the black hole mass, $M=U(S)$ gives the first law in the form

$$
d M=T d S
$$

\footnotetext{
E-mail: bdolan@thphys.nuim.ie
} 
Again for a Schwarzschild black hole, with radius $r_{h}=2 M$ and event horizon area $A=16 \pi M^{2}$, (3) is satisfied only if

$$
S=\frac{1}{4} \frac{A}{\ell_{P l}^{2}},
$$

thus fixing the constant of proportionality in (1) to be $1 / 4$.

More generally, for a rotating black hole, we would have $M=U(S, J)$, with $J$ the angular momentum. The first law then reads

$$
d M=T d S+\Omega d J
$$

where $\Omega$ is the angular velocity. An electric charge is also easily accommodated.

In ordinary thermodynamics the internal energy $U(S, V, n)$, for $n$ moles of a gas in a volume $V$ in $d$ dimensions, is a function of the extensive variables $S, V$ and $n . U$ is also itself extensive so simple scaling implies that

$$
\begin{array}{rlr}
\lambda^{d} U(S, V, n) & =U\left(\lambda^{d} S, \lambda^{d} V, \lambda^{d} n\right) \\
\Rightarrow \quad U & =S \frac{\partial U}{\partial S}+V \frac{\partial U}{\partial V}+n \frac{\partial U}{\partial n} \quad \text { Euler equation } \\
\Rightarrow \quad U & =S T-V P+n \mu \quad(\mu=\text { chemical potential }) \\
\Rightarrow \quad G & =U+V P-S T=n \mu,
\end{array}
$$

where $G(T, P, \mu)$ is the Gibbs free energy. This last equation, stating that $G=n \mu$, is the famous GibbsDuhem relation.

In contrast the dimensionful thermodynamic variables for a black hole in $D$ space-time dimensions, with angular momenta $J_{i}$ (there is more than one angular momentum in $D>4$ ), scale as ${ }^{1}$

$$
S \rightarrow \lambda^{D-2} S, \quad J_{i} \rightarrow \lambda^{D-2} J_{i}, \quad M \rightarrow \lambda^{D-3} M
$$

so

$$
\begin{aligned}
\lambda^{D-3} M\left(S, J_{i}\right) & =M\left(\lambda^{D-2} S, \lambda^{D-2} J_{i}\right) \\
(D-3) M & =(D-2) S \frac{\partial M}{\partial S}+(D-2) J_{i} \frac{\partial M}{\partial J_{i}} \\
\Rightarrow \quad(D-3) M & =(D-2) S T+(D-2) J_{i} \Omega_{i} .
\end{aligned}
$$

Indeed this relation, known as the Smarr relation [3], can be verified for Myers-Perry solutions [4] of Einstein's equation corresponding to asymptotically flat rotating black holes.

However (5) in general fails when a non-zero cosmological constant, $\Lambda$, is included and the solution becomes asymptotically de Sitter or anti-de Sitter. We shall focus here on the thermodynamically better understood anti-de Sitter case, $\Lambda<0 .^{2}$

A solution to this problem was suggested in [6]: to include $\Lambda$ as a thermodynamic variable which scales as $\Lambda \rightarrow \lambda^{-2} \Lambda$. This modifies the Smarr relation to

$$
\begin{aligned}
\lambda^{D-3} M\left(S, \Lambda, J_{i}\right) & =M\left(\lambda^{D-2} S, \lambda^{-2} \Lambda, \lambda^{D-2} J_{i}\right) \\
(D-3) M & =(D-2) S \frac{\partial M}{\partial S}-2 \Lambda \frac{\partial M}{\partial \Lambda}+(D-2) J_{i} \frac{\partial M}{\partial J_{i}} \\
\Rightarrow \quad(D-3) M & =(D-2) S T-2 \Lambda \Theta+(D-2) J_{i} \Omega_{i} .
\end{aligned}
$$

$1 M$ here is the ADM mass of the black hole.

2 The thermodynamic interpretation of space-times with $\Lambda>0$ has a number of difficulties, not least the existence of two physically relevant event horizons, the cosmological and the black hole horizons, and hence in general two different Hawking temperatures. Nevertheless some progress can be made by making extra assumptions [5] that are not necessary for $\Lambda \leq 0$. 
where $\Theta:=\left.\frac{\partial M}{\partial \Lambda}\right|_{S, J}$. This relation is satisfied by all known black hole solutions in Einstein gravity, be they asymptotically flat, anti-de Sitter or de Sitter.

The idea of considering $\Lambda$ to be a variable, rather than a fixed constant, as Einstein originally envisioned, goes back 30 years to [7].

\section{Pressure and enthalpy}

With this philosophy in mind we change the notation and, motivated by the physical interpretation of $\Lambda$ as a pressure, define

$$
P=-\frac{\Lambda}{8 \pi} \text {. }
$$

The mass then depends on $P$ and is most naturally interpreted as the enthalpy, $H(S, P, J)$, of the thermodynamic system [6],

$$
M=H(S, P, J) .
$$

The internal energy, $U(S, V, J)$, is the Legendre transform of the enthalpy,

$$
U=H-P V
$$

where $V:=\frac{\partial M}{\partial P}$ is a thermodynamic volume. The first Law of thermodynamics now reads

$$
d U=T d S-P d V+\Omega d J .
$$

Note that the definition of the thermodynamic volume here does not have any a priori geometrical interpretation.

As stated earlier, we shall focus mostly on negative $\Lambda$. A Penrose process for an asymptotically anti-de Sitter electrically neutral black hole, based on Eq. (6), can be up to 52\% efficient as compared to only $29 \%$ for $\Lambda=0$, [8].

In $D$-dimensions the thermodynamic volume for a rotating black hole evaluates to [9]

$$
V=\frac{1}{3}\left(r_{h} A+\frac{4 \pi J^{2}}{M}\right),
$$

where again $A$ is area of the event horizon and $r_{h}$ the radius. This formula is correct for both asymptotically flat and asymptotically AdS black holes, the difference being encoded in how $r_{h}$ depends on $J, M$ and $\Lambda$. It is curious that this agrees with the naïve geometric volume when $J=0$, but this seems to be just co-incidence. Indeed a geometric volume is not well defined, not least because the radial Schwarzschild co-ordinate is a time-like co-ordinate inside the event horizon.

There is a subtlety here in that, for $J=0$, the volume and the entropy are no longer independent. This is a consequence of the fact that, when $J=0$, the enthalpy $H(S, P, 0)$ is linear in $P$, [8], and hence the Legendre transform on $P$ is not invertible in this limit. The solution is simple: calculate all thermodynamic properties with $J \neq 0$ and only then study the non-rotating case by letting $J \rightarrow 0$.

The formula for $V$ in $D>4$ was given in [9] and involves all the angular momenta $J_{i}$, but does not have quite such an elegant expression in terms of the mass.

\section{Equation of state, compressibility and the speed of sound}

The equation of state exhibits a critical point. This is most easily studied in the 4-dimensional case where there is only one angular momentum and, for $J \neq 0$, we can use $J$ to scale $P, V$ and $T$ to be dimensionless. 
The critical point is then at, [8],

$$
(P J)_{\text {crit }} \approx 0.002857,(T \sqrt{J})_{\text {crit }} \approx 0.04175,\left(\frac{V}{J^{3 / 2}}\right)_{c r i t} \approx 115.8
$$

To study the universality properties of the critical point, we define the reduced variables

$$
t:=\frac{T-T_{c}}{T_{c}}, \quad v:=\frac{V-V_{c}}{V_{c}}, \quad p:=\frac{P-P_{c}}{P_{c}}
$$

In terms of which the equation of state can be expanded about the critical point. The result is $[10,11]$

$$
p=2.42 t-0.81 t v-0.21 v^{3}+o\left(t^{2}, t v^{2}, v^{4}\right) .
$$

This should be compared with the corresponding expansion for the van der Waals gas: $p=4 t-6 t v-$ $\frac{3}{2} v^{3}+o\left(t^{2}, t v^{2}, v^{4}\right)$. Although the co-efficients differ the analytic structure is the same implying that the critical exponents are the same (mean field), putting black holes in the same university class as the van der Waals gas. A van der Waals analogy for an electrically charge, non-rotating black hole, in which the electric charge played the rôle of the volume, was first made in [12].

Once it is known how the volume depends on pressure it is possible to define a compressibility for black holes. Both isothermal and adiabatic compressibility of 4-dimensional black holes were calculated in [13]. The adiabatic compressibility

$$
\kappa=-\left.\frac{1}{V} \frac{\partial V}{\partial P}\right|_{S, J}
$$

of asymptotically AdS Myers-Perry black holes in D-dimensions was calculated in [14]. The expression for general negative $\Lambda$ is not very illuminating, but has an elegant form in the asymptotically flat limit, $\Lambda \rightarrow 0$. In this limit, it is useful to render the angular momenta dimensionless using the entropy and define

$$
\mathcal{J}_{i}:=\frac{2 \pi J_{i}}{S}
$$

There are $\frac{D-2}{2}$ angular momenta $\mathcal{J}_{i}$ in even $D$ and $\frac{D-1}{2}$ in odd $D$. The $\mathcal{J}_{i}$ are constrained though, positivity of the temperature requires

$$
T \geq 0 \quad \Rightarrow \quad \sum_{i} \frac{1}{1+\mathcal{J}_{i}^{2}} \geq \begin{cases}\frac{1}{2} & \text { even } D \\ 1 & \text { odd } D .\end{cases}
$$

With this proviso, the $\Lambda \rightarrow 0$ adiabatic compressibility corresponding to asymptotically flat MyersPerry black hole in $D$ dimensions is, [14],

$$
\kappa=\frac{16 \pi r_{h}^{2}}{(D-1)(D-2)^{2}}\left\{\frac{(D-2) \sum_{i} \mathcal{J}_{i}^{4}-\left(\sum_{i} \mathcal{J}_{i}^{2}\right)^{2}}{D-2+\sum_{i} \mathcal{J}_{i}^{2}}\right\} .
$$

The compressibility is non-negative, $0 \leq \kappa<\infty$.

An example is shown in Fig. 1 of the compressibility in $D=6$, where there are two angular momenta for $S O(5), J_{1}$ and $J_{2}$. The figure on the left shows the adiabatic compressibility as a function of $\mathcal{J}_{1}$ and $\mathcal{J}_{2}$ while the right-hand figure shows the region of $\mathcal{J}$-space allowed by (9), $T>0$ inside the hyperbolae.

Defining $\rho:=\frac{M}{V}$, a thermodynamic speed of sound can be obtained from

$$
c_{s}^{2}=\left.\frac{\partial P}{\partial \rho}\right|_{S, J} \quad \Rightarrow \quad c_{s}^{-2}=1+\kappa \rho .
$$




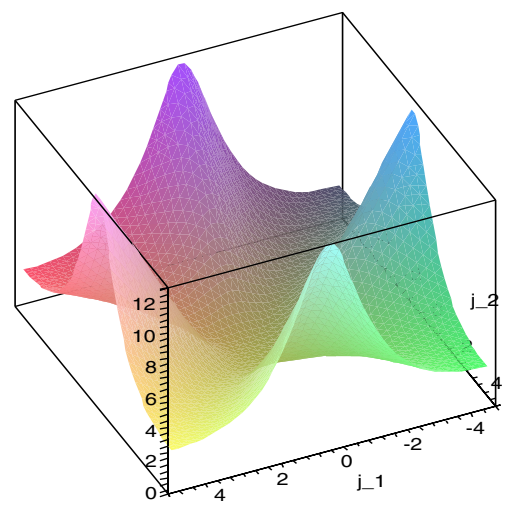

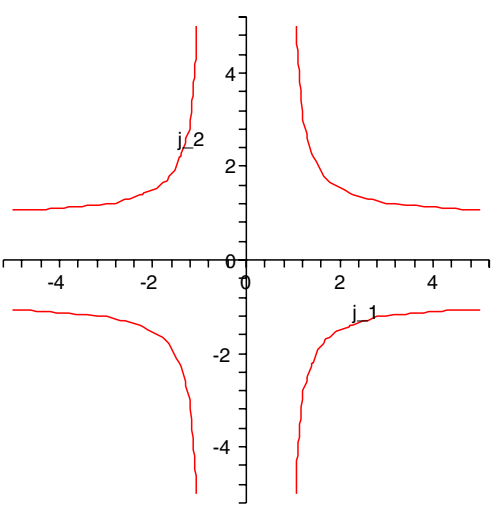

Fig. 1 Compressibility of an asymptotically flat black hole rotating in $6 D$ (left figure). Region of positive $T$ (inside hyperbolae in right figure).

Using (10), the speed of sound for an asymptotically flat Myers-Perry black hole can be written purely in terms of Casimirs of $S O(D-1)$,

$$
c_{s}^{2}=\frac{1}{(D-2)} \frac{\left(D-2+\sum_{i} \mathcal{J}_{i}^{2}\right)^{2}}{\left(D-2+2 \sum_{i} \mathcal{J}_{i}^{2}+\sum_{i} \mathcal{J}_{i}^{4}\right)} .
$$

Note that $\frac{1}{D-2} \leq c_{s}^{2} \leq 1$, with $c_{s}^{2}=1$ for non-rotating (incompressible) black holes. For example $c_{s}^{2}$ in 6 -dimensions is shown in Fig. 2

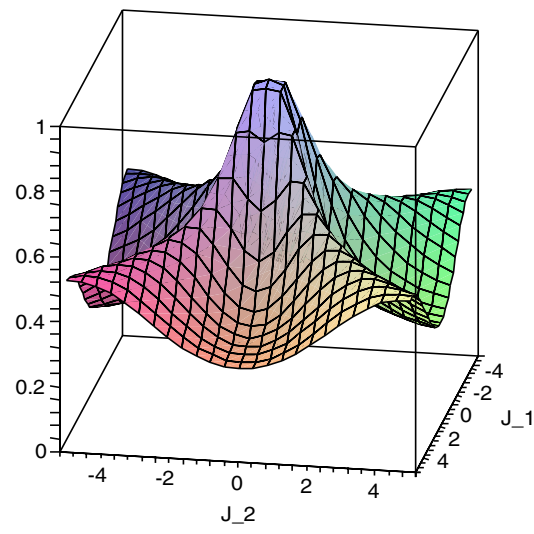

Fig. 2 Speed of sound for an asymptotically flat black hole in $6 D$.

Expressions for the adiabatic compressibility and the speed of sound for asymptotically AdS MyersPerry black holes are given in [14].

\section{Conclusions}

When the cosmological constant is incorporated into the thermodynamic framework of black holes, the Smarr relation requires modification in a way that makes it natural to interpret the black hole mass as the enthalpy, $H\left(S, P, J_{i}\right)$, of the system. For electrically neutral, rotating, black holes

$$
d M=d H=T d S+V d P+\Omega_{i} J_{i}
$$

and the first law must be modified to include a $P d V$ term

$$
d U=T d S-P d V+\Omega_{i} J_{i},
$$

where $U=H-P V$ is a function of $S, V$ and $J_{i}$. 
The Hawking temperature is

$$
T=\left(\frac{\partial H}{\partial S}\right)_{P}
$$

and the "thermodynamic" volume is defined to be

$$
V=\left(\frac{\partial H}{\partial P}\right)_{T} .
$$

The thermodynamics is unambiguous for negative $\Lambda$ in which case the $P d V$ term in the first law can make Penrose processes more efficient in asymptotically AdS space-times than in asymptotically flat spacetimes. The equation of state is of the van der Waals type, with a second order phase transition and mean field exponents.

With the pressure and volume known the compressibility can be calculated and it is positive, $0 \leq \kappa<$ $\infty$, with $\kappa \rightarrow \infty$ for some $J_{i} \rightarrow \infty$ reflecting the instability of ultra-spinning black-holes. A speed of sound can also be defined and it lies withing the physically acceptable regime $\frac{1}{D-2} \leq c_{s}^{2} \leq 1$ for asymptotically flat Myers-Perry black holes in $D$ space-time dimensions.

\section{References}

[1] J. D. Bekenstein, Lett. Nuovo Cimento 4, 737 (1972);

J. D. Bekenstein, Phys. Rev. D 7, 2333 (1973).

[2] S. W. Hawking, Nature 248, 30 (1974);

S. W. Hawking, Commun. Math. Phys. 43, 199 (1975);

S. W. Hawking, Phys. Rev. D 13, 191 (1976).

[3] L. Smarr, Phys. Rev. Lett. 30, 71 (1973).

[4] R. C. Myers and M. J. Perry, Ann. Phys. 172, 304 (1986).

[5] B. P. Dolan, D. Kastor, D. Kubizn̆ák, R. B. Mann, and J. Traschen, Phys. Rev. D 87, 104017 (2013), [arXiv:1301.5926].

[6] D. Kastor, S. Ray, and J. Traschen, Class. Quantum Gravity 26, 195011 (2009), [arXiv:0904.2765].

[7] M. Henneaux and C. Teitelboim, Phys. Lett. 143B, 415 (1984);

M. Henneaux and C. Teitelboim, Phys. Lett. 222B, 195 (1989);

C. Teitelboim, Phys. Lett. 158B, 293 (1985).

[8] B. P. Dolan, Class. Quantum Gravity 28, 235017 (2011), [arXiv:1106.6260].

[9] M. Cvetic, G. W. Gibbons, D. Kubiznák, and C. N. Pope, Phys. Rev. D 84, 024037 (2011), [arXiv:1012.2888].

[10] S. Gunasekaran, D. Kubiznák, and R. B. Mann, J. High Energy Phys. 1211, 110 (2012), [1208.6251 [hep-th]].

[11] B. P. Dolan, Where is the PdV Term in the First Law of Black Hole Thermodynamics? In: Open Questions in Cosmology, edited by G. J. Olmo (InTech, 2012), [arXiv:1209.1272].

[12] A. Chamblin, R. Emparan, C. V. Johnson, and R. C. Myers, Phys. Rev. D 60, 064018 (1999), [arXiv:hepth/9902170];

A. Chamblin, R. Emparan, C. V. Johnson, and R. C. Myers, Phys. Rev. D 60, 104026 (1999), [arXiv:hepth/9904197].

[13] B. P. Dolan, Phys. Rev. D 84, 127503 (2011), [arXiv:1109.0198].

[14] B. P. Dolan, Class. Quantum Gravity 31, 035022 (2014), [arXiv:1308.5403]. 